\title{
FIRST BOUNDS ON THE VERY HIGH ENERGY $\gamma$-RAY EMISSION FROM ARP 220
}

J. Albert,${ }^{1}$ E. Aliu, ${ }^{2}$ H. Anderhub ${ }^{3}$ P. Antoranz, ${ }^{4}$ A. Armada,${ }^{2}$ C. Baixeras, ${ }^{5}$ J. A. Barrio, ${ }^{4}$ H. Bartko, ${ }^{6}$ D. Bastieri, ${ }^{7}$ J. Becker, ${ }^{8}$ W. Bednarek, ${ }^{9}$ K. Berger, ${ }^{1}$ C. Bigongiari, ${ }^{7}$ A. Biland, ${ }^{3}$ R. K. Bock, ${ }^{6,7}$ P. Bordas, ${ }^{10}$ V. Bosch-Ramon, ${ }^{10}$ T. Bretz, ${ }^{1}$ I. Britvitch, ${ }^{3}$ M. Camara, ${ }^{4}$ E. Carmona, ${ }^{6}$ A. Chilingarian, ${ }^{11}$ S. Ciprini, ${ }^{12}$ J. A. Coarasa, ${ }^{6}$ S. Commichau, ${ }^{3}$ J. L. Contreras, ${ }^{4}$ J. Cortina, ${ }^{2}$ V. Curtef, ${ }^{8}$ V. Danielyan, ${ }^{11}$ F. Dazzi, ${ }^{7}$ A. De Angelis, ${ }^{13}$ R. De los Reyes, ${ }^{4}$ B. De Lotto, ${ }^{13}$ E. Domingo-Santamaría, ${ }^{2}$ D. Dorner, ${ }^{1}$ M. Doro, ${ }^{7}$ M. Errando, ${ }^{2}$ M. Fagiolini, ${ }^{14}$ D. Ferenc, ${ }^{15}$ E. Fernández, ${ }^{2}$ R. Firpo, ${ }^{2}$ J. Flix, ${ }^{2}$ M. V. Fonseca, ${ }^{4}$ L. Font,${ }^{5}$ M. Fuchs,${ }^{6}$ N. Galante, ${ }^{6}$ M. Garczarczyk, ${ }^{6}$ M. Gaug, ${ }^{7}$ M. Giller, ${ }^{9}$ F. Goebel ${ }^{6}$ D. Hakobyan, ${ }^{11}$ M. Hayashida, ${ }^{6}$ T. Hengstebeck, ${ }^{16}$ D. Höhne, ${ }^{1}$ J. Hose, ${ }^{6}$ C. C. Hsu, ${ }^{6}$ P. Jacon, ${ }^{9}$ T. Jogler, ${ }^{6}$ O. Kalekin, ${ }^{16}$ R. Kosyra, ${ }^{6}$ D. Kranich, ${ }^{3}$ R. Kritzer, ${ }^{1}$ A. Laille, ${ }^{15}$ P. Liebing, ${ }^{6}$ E. Lindfors, ${ }^{12}$ S. Lombardi, ${ }^{7}$ F. Longo, ${ }^{13}$ J. López, $^{2}$ M. López, ${ }^{4}$ E. Lorenz, ${ }^{3,6}$ P. Majumdar, ${ }^{6}$ G. Maneva, ${ }^{17}$ K. Mannheim, ${ }^{1}$ O. Mansutti, ${ }^{13}$ M. Mariotti, ${ }^{7}$ M. Martínez, ${ }^{2}$ D. Mazin, ${ }^{6}$ C. Merck, ${ }^{6}$ M. Meucci, ${ }^{14}$ M. Meyer, ${ }^{1}$ J. M. Miranda, ${ }^{4}$ R. Mirzoyan, ${ }^{6}$ S. Mizobuchi, ${ }^{6}$ A. Moralejo, ${ }^{2}$ K. Nilsson, ${ }^{12}$ J. Ninkovic, ${ }^{6}$ E. Oña-Wilhelmi, ${ }^{2}$ N. Otte, ${ }^{6}$ I. Oya, ${ }^{4}$ D. Paneque, ${ }^{6}$ R. Padeletti, ${ }^{14}$ J. M. Paredes, ${ }^{10}$ M. Pasanen, ${ }^{12}$

D. Pascoli,${ }^{7}$ F. Pauss ${ }^{3}$ R. Pegna,${ }^{14}$ M. Persic,${ }^{13,18}$ L. Peruzzo, ${ }^{7}$ A. Piccioli, ${ }^{14}$ M. Poller, ${ }^{1}$ N. Puchades, ${ }^{2}$ E. Prandini, ${ }^{7}$ A. Raymers, ${ }^{11}$ W. Rhode, ${ }^{8}$ M. Ribó, ${ }^{10}$ J. Rico, ${ }^{2}$ M. Rissi, ${ }^{3}$ A. Robert,${ }^{5}$ S. RüGamer, ${ }^{1}$

A. Saggion, ${ }^{7}$ A. Sánchez, ${ }^{5}$ P. Sartori, ${ }^{7}$ V. Scalzotto, ${ }^{7}$ V. Scapin, ${ }^{7}$ R. Schmitt, ${ }^{1}$ T. Schweizer, ${ }^{6}$ M. Shayduk, ${ }^{6,16}$ K. Shinozaki, ${ }^{6}$ S. N. Shore, ${ }^{19}$ N. Sidro, ${ }^{2}$ A. Sillanpää, ${ }^{12}$ D. Sobczynska, ${ }^{9}$

A. Stamerra, ${ }^{14}$ L. S. Stark, ${ }^{3}$ L. Takalo, ${ }^{12}$ P. Temnikov, ${ }^{17}$ D. Tescaro, ${ }^{2}$ M. Teshima, ${ }^{6}$ N. Tonello, ${ }^{6}$

D. F. Torres, ${ }^{2,20}$ N. Turini, ${ }^{14}$ H. Vankov, ${ }^{14}$ V. Vitale,${ }^{13}$ R. M. Wagner, ${ }^{6}$ T. Wibig, ${ }^{9}$

W. Wittek, ${ }^{6}$ R. Zanin, ${ }^{2}$ AND J. Zapatero ${ }^{5}$

Received 2006 July 28; accepted 2006 November 21

\begin{abstract}
Using the Major Atmospheric Gamma Imaging Cerenkov Telescope (MAGIC), we have observed the nearest ultraluminous infrared galaxy, Arp 220, for about $15 \mathrm{hr}$. No significant signal was detected within the dedicated amount of observation time. The first upper limits to the very high energy $\gamma$-ray flux of Arp 220 are herein reported and compared with theoretical expectations.
\end{abstract}

Subject headings: gamma rays: observations

Online material: color figure

\section{INTRODUCTION}

The large masses of dense interstellar gas and the enhanced number densities of supernova remnants and massive young stars present in starburst galaxies suggest that they might emit $\gamma$-ray luminosities orders of magnitude greater than normal galaxies and be related to the production of cosmic rays (see, e.g., Torres et al. 2004; Torres \& Anchordoqui 2004). Of the taxonomy of starbursts, a place of privilege is given to luminous and ultraluminous infrared galaxies [LIRGs and ULIRGs; defined as having $\log \left(L_{\mathrm{IR}} / L_{\odot}\right)>11$ and 12 , respectively; see Sanders \& Mirabel

\footnotetext{
1 Universität Würzburg, Würzburg, Germany.

2 Institut de Física d'Altes Energies, Bellaterra (Barcelona), Spain.

3 ETH Zurich, Switzerland.

4 Universidad Complutense, Madrid, Spain.

5 Universitat Autònoma de Barcelona, Bellaterra, Spain.

6 Max-Planck-Institut für Physik, München, Germany.

7 Università di Padova and INFN, Padova, Italy.

8 Universität Dortmund, Dortmund, Germany.

9 University of Łódź, Lodz, Poland.

10 Universitat de Barcelona, Barcelona, Spain.

11 Yerevan Physics Institute, Yerevan, Armenia.

12 Tuorla Observatory, Turku University, Piikkiö, Finland.

13 Università di Udine, and INFN Trieste, Udine, Italy.

14 Università di Siena, and INFN Pisa, Siena, Italy.

15 University of California, Davis, CA.

16 Humboldt-Universität zu Berlin, Berlin, Germany.

17 Institute for Nuclear Research and Nuclear Energy, Sofia, Bulgaria.

18 INAF/Osservatorio Astronomico and INFN Trieste, Trieste, Italy.

19 Università di Pisa, and INFN Pisa, Pisa, Italy.

20 ICREA and Institut de Cienciès de l'Espai, IEEC-CSIC, Bellaterra, Spain. Corresponding author: dtorres@ieec.uab.es.
}

(1996) for a review about these objects]. Such star-forming environments emit a large amount of infrared (IR) radiation because of the abundant dust molecule reprocessing of stellar ultraviolet (UV) photons.

Therefore, the IR luminosity, $L_{\mathrm{IR}}$, of a galaxy can be (but not always) an indication of star formation taking place in it. LIRGs are the dominant population of extragalactic objects in the local universe $(z<0.3)$ at bolometric luminosities above $L>10^{11} L_{\odot}$, and ULIRGs are in fact the most luminous local objects. Most ULIRGs appear to be recent galaxy mergers in which much of the gas of the colliding objects has fallen into a common center (typically less than $1 \mathrm{kpc}$ in extent), triggering a huge starburst (e.g., Sanders et al. 1988; Melnick \& Mirabel 1990). The size of the inner regions of ULIRGs, where most of the gas is found, can be as small as a few hundred parsecs and populated with dense molecular environments (e.g., Gao \& Solomon 2004a, 2004b) that make them prone to having large star formation events and cosmic-ray densities. However, no LIRG, ULIRG, or any other starburst galaxy has been detected in $\gamma$-rays, not even at the EGRET energy range above $100 \mathrm{MeV}$. Upper limits were imposed for M82 and NGC 253, the two nearest starbursts, as well as for many LIRGs (Torres et al. 2004; Cillis et al. 2005). At higher energies, the High Energy Stereoscopic System (H.E.S.S.) has recently reported upper limits for NGC 253 (Aharonian et al. 2005) and we are not aware of any limit reported yet for Arp 220.

Arp 220 is the nearest ULIRG (located at about $72 \mathrm{Mpc}$ ) and the best studied. A complete multiwavelength modeling from radio to $\mathrm{TeV} \gamma$-rays was presented by Torres (2004), where an extensive description on the observational knowledge on this object 

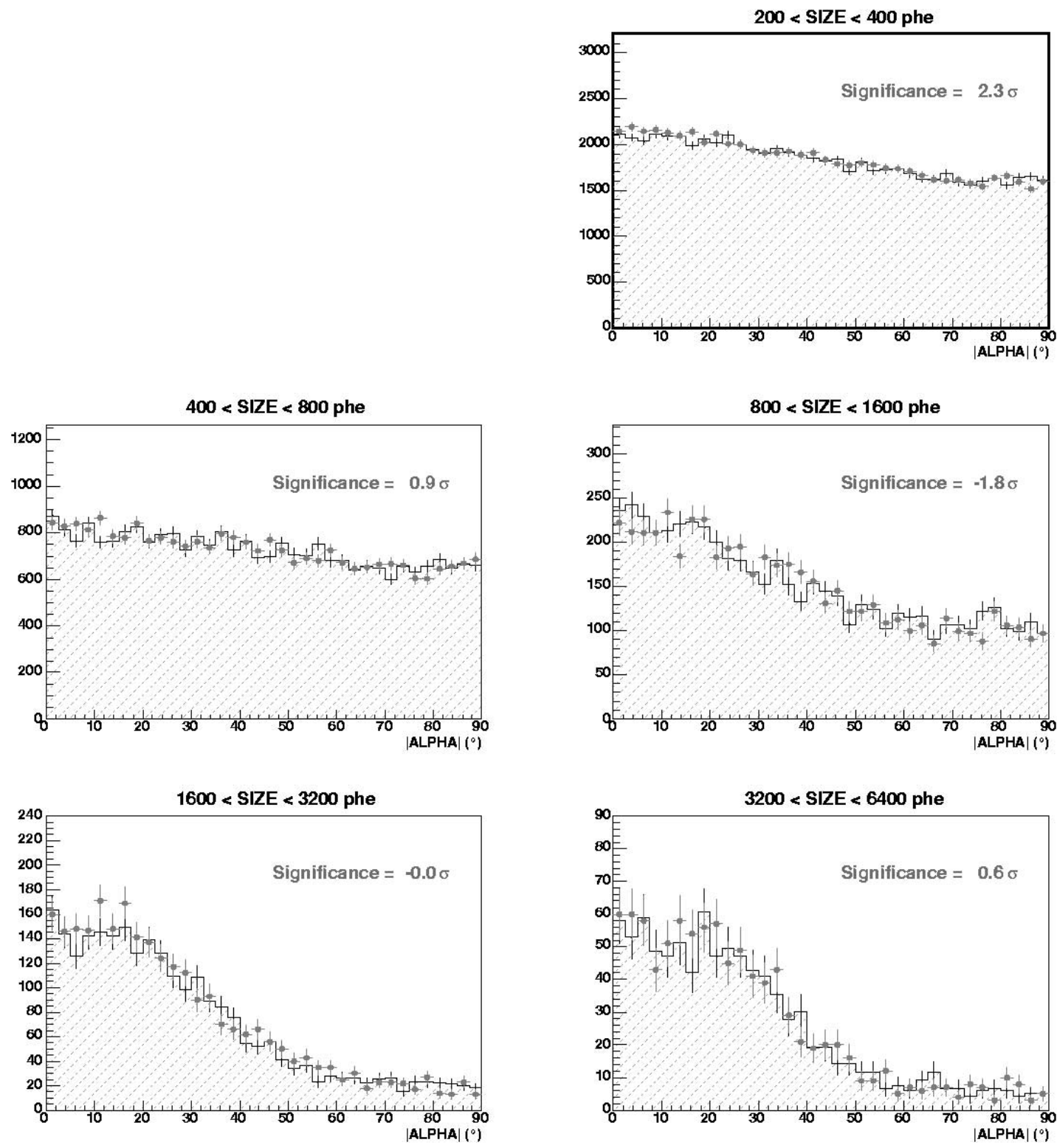

FIG. 1.-ALPHA plots for the Arp 220 data, separated in bins of SIZE. [See the electronic edition of the Journal for a color version of this figure.]

can also be found. Arp 220 possesses the record of being the object with the highest directly measured supernova explosion rate known, with recent measurements placing it at an outrageous $4 \pm 2$ per year (Lonsdale et al. 2006). Such a high supernova explosion rate emphasizes the quality of Arp 220 as a possible $\gamma$-ray target.

\section{OBSERVATIONS}

MAGIC (see, e.g., Baixeras et al. 2004; Cortina et al. 2005 for a detailed description) is a single-dish imaging air Cerenkov telescope. Located on the Canary Island of La Palma (N28.8, $\mathrm{W} 17.8^{\circ}$, $2200 \mathrm{~m}$ above sea level), the telescope has a $17 \mathrm{~m}$ diameter mirror, and it is equipped with a 576 pixel $3.5^{\circ}$ field-of-view photomultiplier (PMT) camera. The analog PMT signals are transported via optical fibers to the trigger electronics and are read

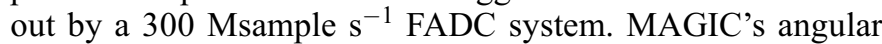
resolution is approximately $0.1^{\circ}$, the energy resolution is about $20 \%$, and the trigger (analysis) threshold is $55(90) \mathrm{GeV}$.

Arp 220's celestial coordinates are (J2000.0) $\alpha=15^{\mathrm{h}} 34^{\mathrm{m}} 57.21^{\mathrm{s}}$, $\delta=+23^{\circ} 30^{\prime} 09.5^{\prime \prime}$, which has allowed MAGIC to always ob- serve it with zenith angle lower than $20^{\circ}$. Observations proceeded from 2005 May to June, for a total time of 947 minutes, of which 925 minutes were selected - after quality checks - for further analysis. MAGIC observations were carried out in the ON-OFF mode, with a similar amount of ON and OFF data being considered for the analysis. This observation mode allows a reliable background estimation. The specific properties of the data sample used, both ON and OFF, can be found in Domingo-Santamaría (2006), in which additional details are available on the quality checks performed and on the cut optimization using an independent Crab sample of data.

The data analysis was carried out using the standard MAGIC analysis and reconstruction software (Wagner et al. 2003), the first step of which involves the calibration of the raw data (Gaug et al. 2005). It follows the general stream presented in Albert et al. (2006a, 2006b, 2006c): After calibration, image cleaning tail cuts of 10 photoelectrons for image core pixels and 5 photoelectrons (boundary pixels) have been applied (see, e.g., Fegan 1997). These tail cuts are accordingly scaled for the larger size of the outer pixels of the MAGIC camera. The camera images are parameterized by 
TABLE 1

Number of Excess and Background Events and the Corresponding Significance and Upper Limits Obtained from the Arp 220 Analysis

\begin{tabular}{|c|c|c|c|c|c|c|c|c|}
\hline $\begin{array}{c}\text { SIZE Bin } \\
\text { (photoelectrons) }\end{array}$ & $\begin{array}{l}\text { Average Energy } \\
\quad(\mathrm{GeV})\end{array}$ & $\begin{array}{l}\text { HADRONNESS } \\
\text { Cut }\end{array}$ & $\begin{array}{l}\text { ALPHA Cut } \\
\quad(\text { deg })\end{array}$ & $\begin{array}{c}\text { Number } \\
\text { Excess Events }\end{array}$ & $\begin{array}{c}\text { Number of } \\
\text { Background Events }\end{array}$ & $\begin{array}{l}N_{\sigma} \\
(\sigma)\end{array}$ & $\begin{array}{l}\text { Crab Rate } \\
\left(\gamma \mathrm{hr}^{-1}\right)\end{array}$ & $\begin{array}{c}3 \sigma \text { Upper Limit } \\
\left(10^{-14} \text { photons } \mathrm{cm}^{-2} \mathrm{~s}^{-1} \mathrm{GeV}^{-1}\right)\end{array}$ \\
\hline $200-400 .$. & 160.7 & 0.20 & 10.00 & 295 & 8343 & 2.3 & 171.3 & 80.14 \\
\hline $800-1600 \ldots \ldots \ldots \ldots . . . .$. & 462.7 & 0.16 & 7.50 & -62 & 707 & -1.8 & 90.2 & 1.00 \\
\hline $1600-3200 \ldots \ldots \ldots . .$. & 773.3 & 0.20 & 6.25 & 7 & 370 & 0.3 & 81.1 & 0.40 \\
\hline $3200-6400 \ldots \ldots \ldots \ldots$ & 1351.8 & 0.18 & 6.25 & 22 & 132 & 1.4 & 33.6 & 0.18 \\
\hline
\end{tabular}

image parameters (Hillas 1985). In this analysis, the random forest method (see Bock et al. 2004; Breiman 2001 for a detailed description) was applied for the $\gamma /$ hadron separation.

The source position-independent image parameters SIZE, WIDTH, LENGTH, and CONC (Hillas 1985) and the third moment of the photoelectron distribution along the major image axis were selected to parameterize the shower images. After the training, the random forest method allows us to calculate a parameter for every event, the HADRONNESS, which is a measure of the probability that the event is not $\gamma$-like. The $\gamma$-sample is defined by selecting showers with a HADRONNESS below a specified value, which is optimized using a sample of Crab data that has been processed with the same analysis stream. An independent sample of Monte Carlo $\gamma$-showers was used to determine the cut efficiency.

Figure 1 shows the ALPHA plots that were obtained for the whole sample of ON and OFF data after applying the HADRONNESS cuts. The ON and OFF ALPHA distributions match reasonably well within fluctuations. No signal above the background level is observed in any of the SIZE bins. Details are given in Table 1; we also give the Crab rate there (obtained with $1.22 \mathrm{hr}$ of data under the same analysis stream applied to Arp 220). The upper limits to the very high energy $\gamma$-ray flux from Arp 220 can then be calculated out of the number of excess and background events, in a similar way to that used in the case of LS I +61 303 (Albert et al. 2006d), using the method by Rolke \& López (2001). The final column of Table 1 summarizes these results.

\section{DISCUSSION AND CONCLUDING REMARKS}

The upper limits imposed to the differential flux of Arp 220 with $15.4 \mathrm{hr}$ of data are above the theoretical curves at all energies (see Fig. 2). ${ }^{21}$ All upper limits are at least about 1 order of magnitude above the curve obtained using the $\delta$-function (Aharonian \& Atoyan 2000) or Kamae et al. (2005) approaches for the protonproton cross-section parameterization. The latter (see the Appendix in Domingo-Santamaría \& Torres 2005 for a detailed discussion) are the most reliable parameterizations of the proton-proton cross section, which is devoid of the intrinsic problems of extrapolating the Blattnig et al. (2000) formulae to high energies. The current results imply that under the same conditions of

\footnotetext{
${ }^{21}$ The theoretical curves are the result of a multiwavelength modeling discussed by Torres (2004) and Torres \& Domingo-Santamaría (2005). From the proton steady state population the computation of the secondary $e^{ \pm}$proceeds considering knock-on interactions and decay of charged pions. The lepton population is allowed to evolve to its steady state, computing the energy losses by synchrotron emission, ionization, bremsstrahlung, inverse Compton, and adiabatic expansion, and their confinement timescale. The radio spectrum is evaluated from the steady electron synchrotron emission, modulated by free-free absorption. Infrared emission from dust is also simulated to describe the observational data. These photons are the seeds of the inverse Compton process. Once the multiwavelength spectrum of the object has been reproduced, the high-energy $\gamma$-ray emission is evaluated through the decay of neutral pions, and the bremsstrahlung and inverse Compton of the steady electron population. Photon absorption is considered to obtain the final predictions of fluxes.
}

telescope performance and sensitivity of the data analysis applied, the amount of observation time needed to be devoted to Arp 220 in order to be able to confirm or reject the predictions from the $\gamma$-ray emission of this object is too high for a detector with a typical duty cycle of about $1000 \mathrm{hr}$ per year, such as MAGIC. Disregarding the fact that Arp 220 is the nearest ULIRG and the galaxy with the largest supernova explosion rate we know, its distance dilutes the putative $\gamma$-ray flux it produces. MAGIC upper limits are consistent with this interpretation and with the theoretical prediction that results from a complete multiwavelength modeling of the object. The first detection of $\gamma$-rays from starburst regions beyond our galaxy has yet to be achieved.

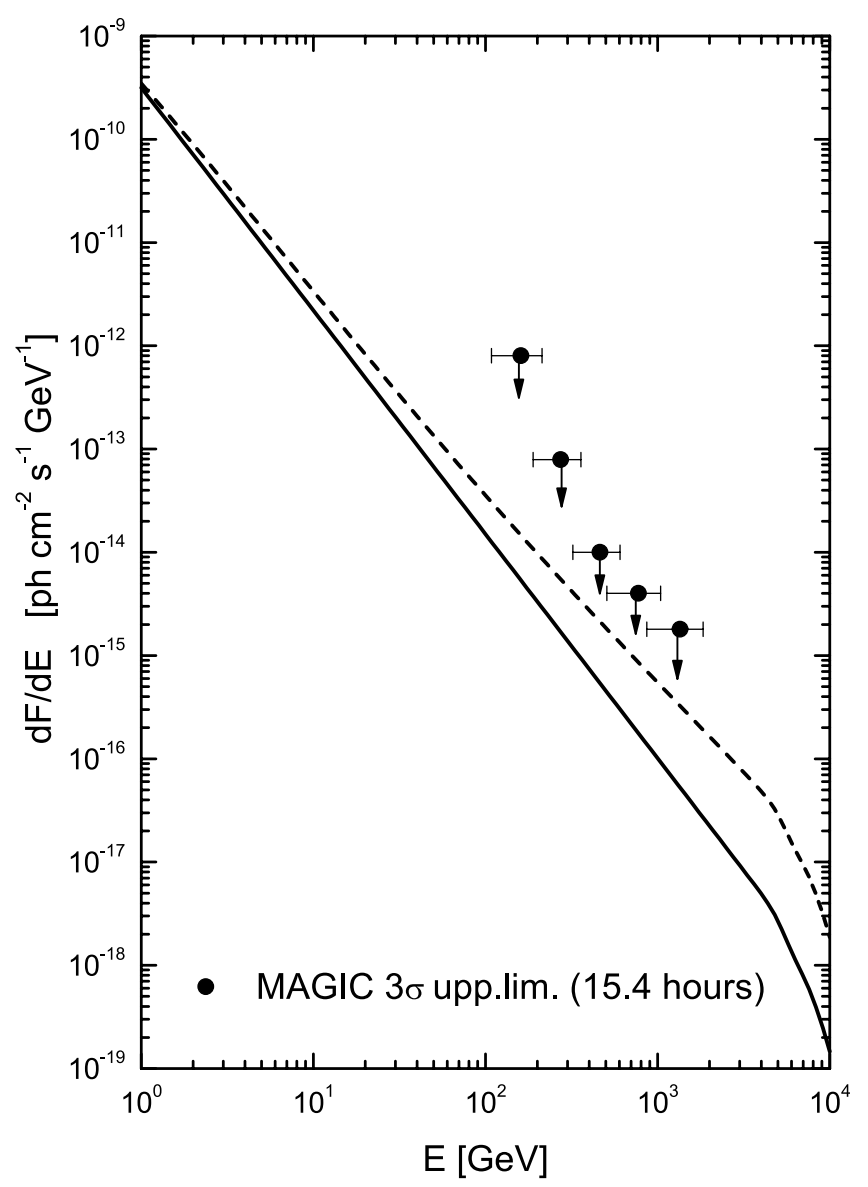

FIG. 2.-MAGIC upper limits to the differential $\gamma$-ray flux of Arp 220. The curves represent the theoretical predictions; the solid line shows the result using the $\delta$-function (Aharonian \& Atoyan 2000) or Kamae et al. (2005) approximations for the proton-proton cross section, whereas the dashed line shows the result using the parameterization proposed by Blattnig et al. (2000), extrapolated to high energies. Theoretical curves are from Torres (2004) and Torres \& Domingo-Santamaría (2005). 
We would like to thank the IAC for the excellent working conditions at the Observatory de los Muchachos in La Palma. The support of the German BMBF and MPG, the Italian INFN and the Spanish CICYT is gratefully acknowledged. This work was also supported by ETH research grant TH 34/04 3 and the Polish MNiI grant 1P03D01028.
Aharonian, F. A., \& Atoyan, A. M. 2000, A\&A, 362, 937

Aharonian, F. A., et al. (the HESS Collaboration). 2005, A\&A, 442, 177

Albert, J., et al. (the MAGIC Collaboration). 2006a, ApJ, 637, L41 2006b, ApJ, 643, L53

2006c, ApJ, 638, L101

2006d, Science, 312, 1771

Baixeras, C., et al. (the MAGIC Collaboration). 2004, Nucl. Instrum. Methods Phys. Res. A, 518, 188

Blattnig, S. R., Swaminathan, S. R., Kruger, A. T., Ngom, M., \& Norbury, J. W. 2000, Phys. Rev. D, 62, 094030

Bock, R. K., et al. 2004, Nucl. Instrum. Methods Phys. Res. A, 516, 511

Breiman, L. 2001, Machine Learning, 45, 5

Bretz, T., \& Wagner, R. (for the MAGIC Collaboration). 2003, in Proc. 28th Int. Cosmic Ray Conf. (Tsukuba), 2947

Cillis, A. N., Torres, D. F., \& Reimer, O. 2005, ApJ, 621, 139

Cortina, J., et al. (the MAGIC Collaboration). 2005, in Proc. 29th Int. Cosmic Ray Conf. (Pune), 5, 359

Domingo-Santamaría, E. 2006, Ph.D. thesis, Univ. Autonoma Barcelona, http:// wwwmagic.mppmu.mpg.de/publications/theses/index.html

Domingo-Santamaría, E., \& Torres, D. F. 2005, A\&A, 444, 403

\section{REFERENCES}

Fegan, D. J. 1997, J. Phys. G, 23, 1013

Gao, Y., \& Solomon, P. M. 2004a, ApJS, 152, 63 2004b, ApJ, 606, 271

Gaug, M., et al. (the MAGIC Collaboration). 2005, in Proc. 29th Int. Cosmic Ray Conf. (Pune), 5, 375

Hillas, A. M. 1985, in Proc. 19th Int. Cosmic Ray Conf. (La Jolla), 3, 445

Kamae, T., Abe, T., \& Koi, T. 2005, ApJ, 620, 244

Lonsdale, C. J., Diamond, P. J., Thrall, H., Smith, H. E., \& Lonsdale, C. J. 2006, ApJ, 647, 185

Melnick, J., \& Mirabel, I. F. 1990, A\&A, 231, L19

Rolke, W. A., \& López, A. M. 2001, Nucl. Instrum. Methods Phys. Res. A, 458,745

Sanders, D. B., \& Mirabel, I. F. 1996, ARA\&A, 34, 749

Sanders, D. B., Soifer, B. T., Elias, J. H., Madore, B. F., Matthews, K., Neugebauer, G., \& Scoville, N. Z. 1988, ApJ, 325, 74

Torres, D. F. 2004, ApJ, 617, 966

Torres, D. F., \& Anchordoqui, L. A. 2004, Rep. Prog. Phys., 67, 1663

Torres, D. F., \& Domingo-Santamaría, E. 2005, Mod. Phys. Lett. A, 20, 2827

Torres, D. F., Reimer, O., Domingo-Santamaría, E., \& Digel, S. 2004, ApJ, 607, L99 